\title{
Letter to the Editor
}

\section{Retinal photoreceptors are apoptosis-competent in the absence of JunD/AP-1}

\section{Dear Editor,}

In the retina, apoptosis is an essential component in retinal development and differentiation, but in addition it is also a major component in human retinal degeneration. ${ }^{1}$ In many animal models for this human disease group, apoptosis is the final common pathway of photoreceptor cell death. ${ }^{2}$ To date, little is known about the molecular mechanisms leading to apoptosis in the retina. Recently, we identified the AP-1 component c-Fos as essential for light-induced photoreceptor apoptosis: non-functional c-Fos completely inhibits lightinduced photoreceptor degeneration in the mouse retina in vivo. $^{3}$ To understand the mechanism of this protection it is crucial to learn more about the role of AP-1 and its constituents in the retina.

AP-1 is a transcription factor complex that may be differentially assembled from members of the Fos (c-Fos, FosB, Fra-1, Fra-2) and Jun (c-Jun, JunD, JunB) family of proteins. ${ }^{4}$ The complex composition of AP-1 determines its biological function by modulating the transcription rate of specific sets of target genes, AP-1 complexes containing cFos exhibiting the strongest transactivation activity. ${ }^{4}$ Activation of AP-1 is involved in apoptotic cell death in a variety of different systems. ${ }^{5-7}$ We have shown recently that in the mouse retina AP-1 complexes mainly consist of C-Fos, JunD and C-Jun. ${ }^{8}$ Interestingly, elevated expression of AP-1 consisting of $\mathrm{c}-\mathrm{Fos} / \mathrm{JunD}$ is also observed during apoptosis in other tissues. ${ }^{7}$

To address the role of Jun proteins in AP-1 complexes during photoreceptor apoptosis, we have used transgenic mice lacking functional JunD in our model of light-induced photoreceptor degeneration. The effect of c-Jun depletion could not be tested since cJun knockouts die at midgestation E12.5. ${ }^{9}$

JunD mutants (Thepot et al, submitted) were bred from heterozygous matings on a mixed C57BL/6xSv129 background (verification of the genotype by PCR analysis) and maintained in a $12: 12 \mathrm{~h}$ light-dark cycle (lights on at $0600 \mathrm{~h}$ ) with 10-20 lux within the cages. Six to ten-weeksold $j u n D^{-1-}$ and $j u n D^{+/+}$mice were dark adapted for $16 \mathrm{~h}$ and the pupils were dilated with cyclopentolate $1.0 \%$ (Alcon Pharmaceuticals, Cham, Switzerland) and phenylephrin 5\% (Ciba Vision, Niederwangen, Switzerland).

Mice were anaesthetized with $\mathrm{CO}_{2}$ and killed by cervical dislocation either before light exposure (dark control) or after $24 \mathrm{~h}$ of darkness following exposure to 15000 lux of diffuse, cool, white fluorescent light for $2 \mathrm{~h}$. Eyes were removed and processed for light microscopy, in situ analysis of DNA strand breaks (TUNEL) or analysis of genomic DNA by gel electrophoresis.

Dark adapted junD ${ }^{+/+}$and $j u n D^{-/-}$mice displayed regular retinal morphology (Figure $1 \mathrm{~A}, \mathrm{a}, \mathrm{b}$ ) suggesting that physiological apoptosis during development occurred in the absence of JunD. After exposure to 15000 lux for $2 \mathrm{~h}$ and a post-exposure time of $24 \mathrm{~h}$ in darkness, both junD ${ }^{+/+}$and jun $D^{-1-}$ mice showed distinct signs of photoreceptor apoptosis (Figure 1A, c,d). Light microscopy revealed condensed photoreceptor nuclei in the outer nuclear layer (ONL), condensed rod inner segments (RIS) and disrupted rod outer segments (ROS). TUNEL staining showed comparable positive labeling of nuclei in the ONL of both groups of animals at this time point (Figure 1B, c,d), whereas the retinas of unexposed $j u n D^{+/+}$and $j u n D^{-/-}$ animals were devoid of signals (Figure 1B, a,b). Similarly, DNA fragmentation was observed in light-exposed $j u n D^{+/+}$ and $j u n D^{-1-}$ animals while the DNA of unexposed controls was intact (Figure 1C). These data indicate that photoreceptor apoptosis is independent of functional JunD.

In the rodent retina, little is known about the specific role of the AP-1 members c-Fos and JunD during the maintenance of retinal function and morphology. Basal cfos expression follows a diurnal rhythm ${ }^{10}$ and is predominantly found in the ONL. ${ }^{11}$ Furthermore, elevated levels of c-fos mRNA in the ONL precede photoreceptor apoptosis in light-induced retinal degeneration ${ }^{3}$ and in several animal models for inherited retinal dystrophy such as the retinal degeneration $(r d)$ and the retinal degeneration slow ( $r d s)$ mouse. ${ }^{12,13}$

In contrast, the distribution pattern of JunD remains unchanged in the normal and the $r d$ mouse with high constitutive levels of JunD in both the Inner Nuclear Layer (INL) and ONL. ${ }^{13}$ Similarly, the low-level expression of cJun in the INL and ONL of the normal mouse retina remains unchanged in the $r d$ mouse. ${ }^{13}$

In conclusion, in this communication we report that JunD is not essential for light-induced apoptosis of photoreceptor cells in the adult mouse retina. Our data further suggest that developmental apoptosis is not affected by the lack of JunD either. Since AP-1 in the mouse retina predominantly consists of c-Fos, c-Jun and JunD, ${ }^{8}$ it is conceivable that, in contrast to c-Fos, the lack of JunD is compensated by other Jun family members. Further studies on other transgenic mice such as an inducible c-Jun mutant on a JunD depleted background are needed to test this hypothesis.

Supported by the Swiss National Science Foundation, No. 31-40791.94, Sandoz Foundation, Basel, Switzerland, Bruppacher Foundation, Zurich, Switzerland and lan and Caroline Leaf and family, Gland, Switzerland. 
A

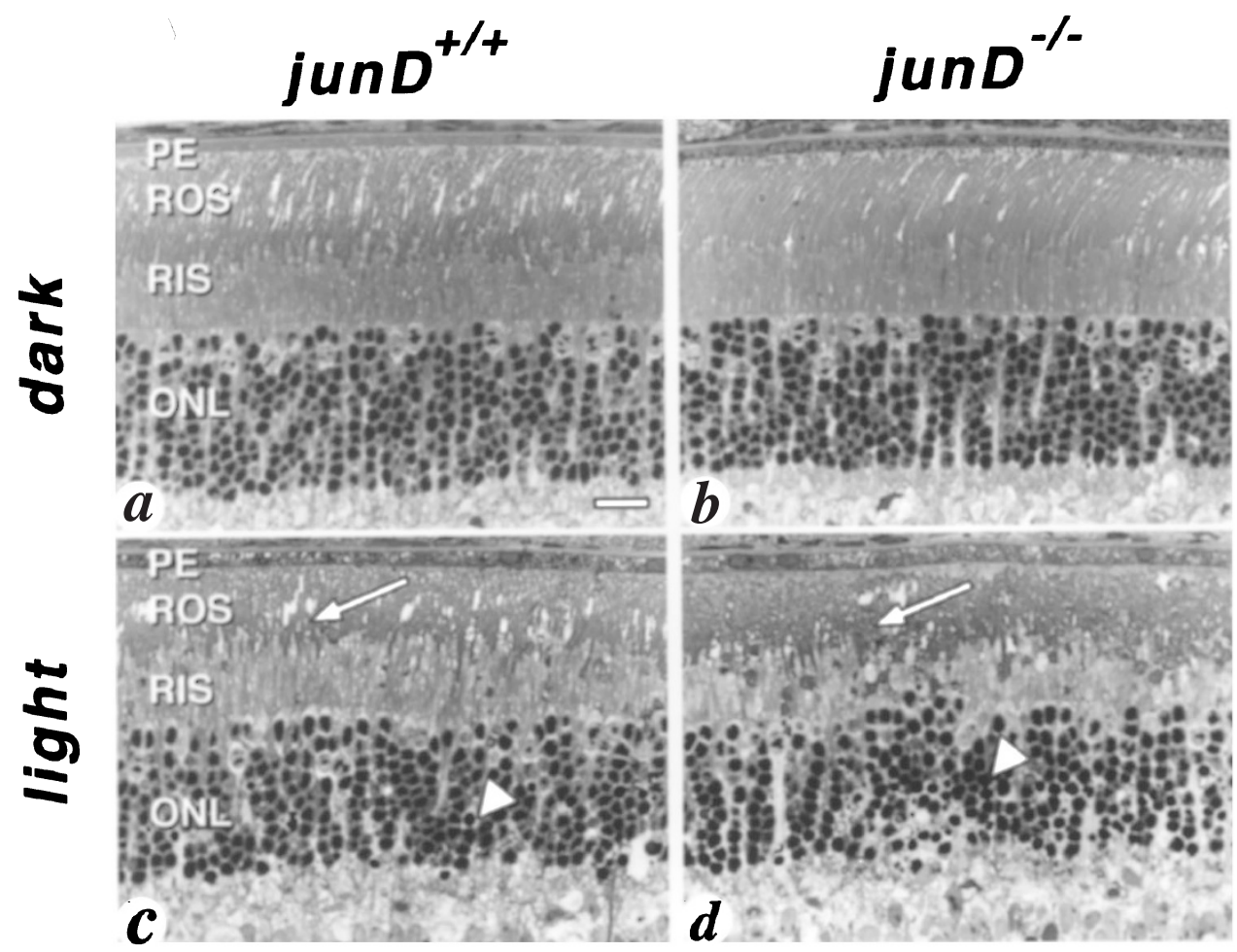

B
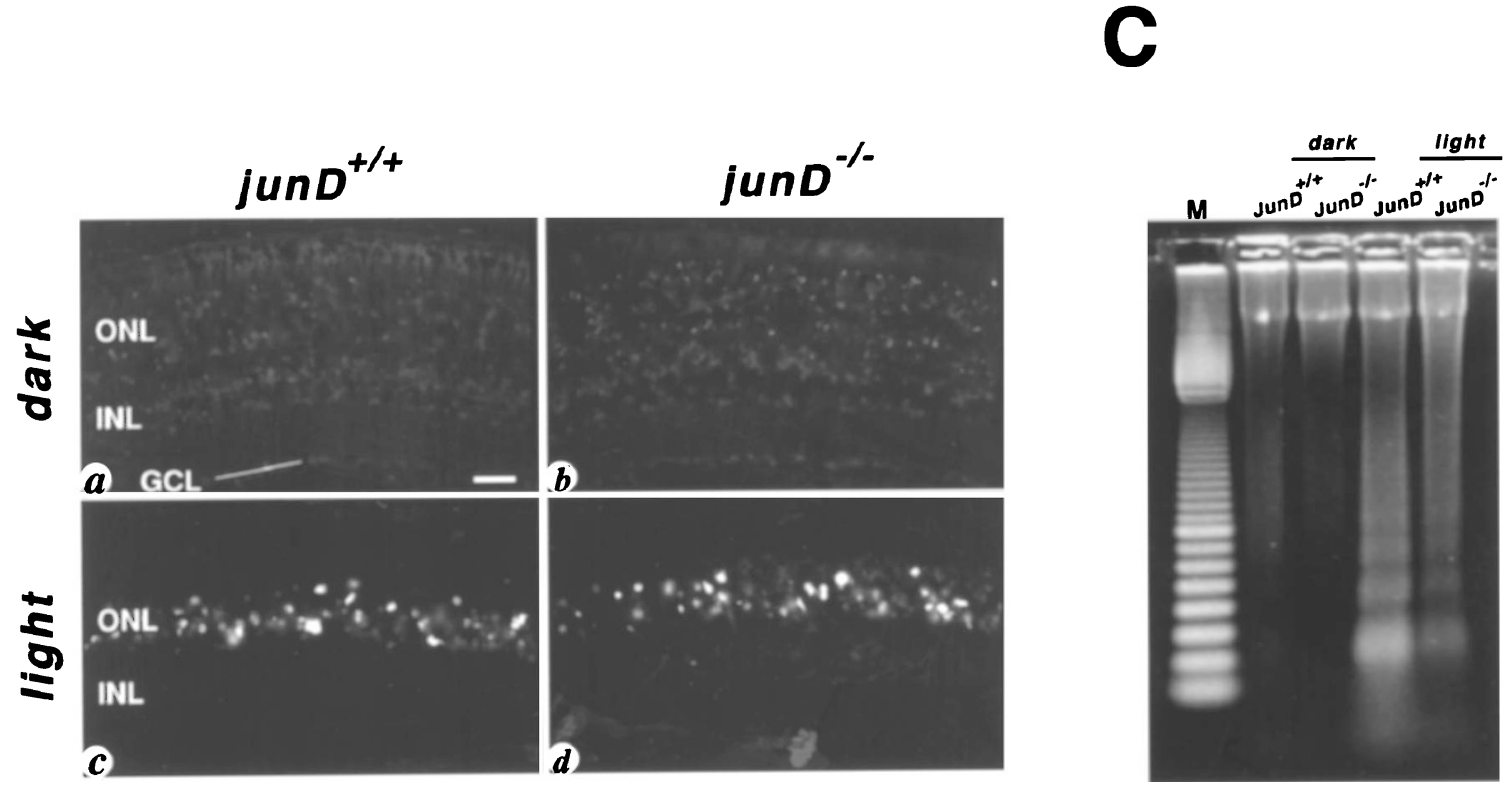

Figure 1 Effect of JunD depletion on light-induced photoreceptor apoptosis. (A) Light microscopy of retinal sections in junD knockout mice and wild-type control littermates. Retinas were analyzed prior to illumination (dark; $a$ and b) or following exposure to white light (light; $c$ and d). Arrowheads show condensed photoreceptor nuclei in the ONL. Arrows show disrupted ROS. Scale bar $20 \mu \mathrm{m}$. For light microscopic analysis, eyes were enucleated, fixed in $2.5 \%$ glutaraldehyde for $12 \mathrm{~h}$, dehydrated and processed as described ${ }^{3}$. (B) Detection of DNA strand breaks in photoreceptor nuclei by in situ nick end-labeling (TUNEL). Retinas of junD knockout mice and of wild-type controls were prepared for TUNEL staining prior to illumination (dark; $a$ and b) or following exposure to white light (light; $c$ and d). Scale bar $100 \mu \mathrm{m}$. Eyes were fixed in $2 \%$ paraformaldehyde for $2 \mathrm{~h}$, dehydrated, embedded in paraffin and stained as described. ${ }^{3}$ (C) DNA fragmentation analysis. Total retinal DNA was prepared ${ }^{3}$ from junD knockout mice and wild-type controls prior to (dark) and after light exposure (light). M, $100 \mathrm{bp} \mathrm{standard}$ 


\section{Farhad Hafezi ${ }^{*, 1}$ Christian Grimm ${ }^{1}$, Andreas Wenzel', Jonathan Weitzman Mathias Abegg ${ }^{1}$, Moshe Yaniv and Charlotte E Remé}

1 Department of Ophthalmology, University Hospital, 8091 Zurich,

Switzerland

${ }^{2}$ Unité des virus oncogenes, Institut Pasteur,

Rue du Dr. Roux, Paris, France ${ }^{*}$ corresponding author: Farhad Hafezi MD,

Department of Ophthalmology, University Hospital, 8091 Zurich,

Switzerland

tel: +4112553719 ,

fax: +4112554385,

E-mail: hafezi@opht.unizh.ch
1. Li ZY and Milam AH. In Retinal Degeneration, Anderson RE, LaVail MM, Hollyfield JG (eds) 1995; Plenum Press: New York, London, pp 1-8

2. Chang GQ, et al. (1993) Neuron 11: 595-605

3. Hafezi F, et al. (1997) Nat. Med. 3: 346-349

4. Curran T and Franza Jr BR (1988) Cell 55: 395-397

5. Ham J, et al. (1995) Neuron 14: 927-939

6. Bossy Wetzel E, et al. (1997) EMBO J. 16: 1695-1709

7. Marti A, et al. (1994) Oncogene 9: 1213-1223

8. Hafezi F, et al. (1999) Vision Res. 39: 2511-2518

9. Johnson RS, et al. (1993) Genes Dev. 7: 1309-1317

10. Nir I and Agarwal N (1993) Brain Res. Mol. Brain Res. 19: 47-54

11. Yoshida K, et al. (1993) Neuron 10: 1049-1054

12. Agarwal N, et al. (1995) Invest. Ophthalmol. Vis. Sci. 36: 2921

13. Rich KA, et al. (1997) J. Neurobiol. 32: 593-612 